\title{
Optimized Sample Preparation for MALDI Mass Spectrometry Analysis of Protected Synthetic Peptides
}

\author{
Audrey M. Schaiberger and Jason A. Moss \\ Roche Colorado Corporation, Boulder Technology Center, Boulder, Colorado, USA
}

The recent development and commercialization of Fuzeon (enfuvirtide) demonstrated that a convergent strategy comprised of both solid- and solution-phase synthetic methodologies presents a viable route for peptide manufacturing on a multi-ton scale. In this strategy, the target sequence is prepared by stepwise solid-phase synthesis of protected peptide fragments, which are then coupled together in the solution-phase to give the full-length sequence. These synthetic methodologies pose a unique challenge for mass spectrometry (MS), as protected peptide intermediates are often marked by poor solubility, structural lability, and low ionization potential. Matrix-assisted laser desorption/ionization (MALDI) MS is uniquely suited to such analytes; however, generalized protocols for MALDI analysis of protected peptides have yet to be demonstrated. Herein, we report an operationally simple sample preparation method for MALDI analysis of protected peptides, which greatly facilitates the collection and interpretation of MS data. In this method, the difficulty in MS analysis of protected peptides has been greatly diminished by use of dithranol as a matrix and $\mathrm{CsCl}$ as an additive, giving rise to intentionally-formed $\mathrm{Cs}^{+}$adducts. With greatly reduced fragmentation, better crystalline morphology, and easier data interpretation, we anticipate that these findings will find utility in peptide process development and manufacturing settings for reaction monitoring, troubleshooting, and quality control. (J Am Soc Mass Spectrom 2008, 19, 614-619) (C) 2008 American Society for Mass Spectrometry

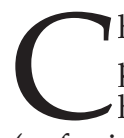
hemical synthesis is now a proven method for peptide manufacturing on a multi-ton scale, having recently been demonstrated by Fuzeon (enfuvirtide), a 36 amino acid residue peptide HIV fusion inhibitor [1,2]. The convergent synthetic strategy developed for this process employs a two-step combination of solid- and solution-phase methodologies. In the first step, solid-phase synthesis is used to prepare the three side-chain-protected fragments (1-16, 17-26, and 27-36), which encompass the full-length 36 amino acid residue sequence. In the second step, these fragments are joined in the solution phase to afford the full-length sequence, which is then deprotected to furnish the final active pharmaceutical ingredient (API). As a result of its high yields and production efficiency, this technology shows promise for a number of other peptide-based compounds in pharmaceutical development. These benefits notwithstanding, the unique physicochemical characteristics of protected peptide intermediates pose a challenge to high-resolution MS analysis, a critical step in analytical monitoring of synthetic processes.

Address reprint requests to Dr. Jason A. Moss, Roche Colorado Corporation, Boulder Technology Center, 2075 North 55th Street, Boulder, CO 80301, USA. E-mail: jason.moss@roche.com
In most cases, the majority-if not all-of the common proteinogenic functional groups are masked during peptide synthesis so that they will not interfere with iterative coupling and deprotection procedures. While indispensable from a synthetic perspective, these protecting groups (e.g., Fmoc, Boc, $t \mathrm{Bu}$, Trt, Pbf) present multiple problems from an analytical perspective. First, protection of reactive functional groups results in abrogation of their ionization potential, rendering MS analysis under mild conditions difficult, if at all possible. Moreover, protected peptides are often only sparingly soluble, and in many cases require aggressive solvents (e.g., fluorinated alcohols, DMSO, DMF) for dissolution, which are incompatible with many MS methods. Finally, even if ionization is accomplished, this is usually accompanied by significant source-induced fragmentation, usually at protected functional groups, with the net result of artifactually "impure" MS data incongruous with the purity of the actual sample.

Among MS methods, MALDI-TOF instruments are uniquely suited to the analysis of protected peptides for several inter-related reasons. Since ionization occurs directly from a solid, dry phase, the MALDI source is ideal for analytes of limited solubility in aqueous buffer systems such as those commonly employed in ESIequipped instruments. Moreover, time-of-flight (TOF) 
analysis is ideal for high MW analytes which, by virtue of intact side-chain protecting groups, will acquire only a single charge; most ion trap or quadrupole mass analyzers have a $m / z$ range limited at 2000-3000 and are therefore of limited utility in detection of singlycharged ions of $>3000$ Da. Finally, MALDI is one of the most tolerant MS techniques with respect to salts and solvents, principally due to its extreme (i.e., femtomole) sensitivity, which allows virtually any analyte solution to be diluted extensively such that contaminant concentrations are reduced to the sub-millimolar level [3].

MALDI sample preparation has been optimized for MS analysis of analytes with unique physicochemical properties; notable examples include polymers [4], phosphopeptides [5], low abundance proteins [6, 7], and oligonucleotides $[8,9]$. However, it has not, to our knowledge, been optimized for the analysis of protected peptides. While this class of compounds was formerly of limited importance, MS analysis of protected peptide intermediates in process development and manufacturing environments has been given new impetus as a result of the recent "renaissance" in convergent peptide synthesis methods for active pharmaceutical ingredient API manufacturing. In the work described herein, we describe the rational optimization, using both empirical and physicochemical criteria, of MALDI sample preparation to provide a generally applicable and operationally simple protocol for in-process analysis of protected peptide synthetic intermediates.

\section{Experimental}

\section{Materials and Reagents}

Fuzeon fragments (FF) 1, 2, and 3 were obtained from Roche Colorado Corporation Manufacturing (Boulder, $\mathrm{CO})$ as in-process sample retains. Fragment sequences and masses are as follows: FF1, Ac-Tyr(tBu)-Thr(tBu)Ser(tBu)-Leu-Ile-His(Trt)-Ser(tBu)-Leu-Ile-Glu(OtBu)Glu(OtBu)-Ser(tBu)-Gln(Trt)-Asn(Trt)-Gln(Trt)-Gln-OH, MW (monoisotopic) 3291.80, (average) 3294.05; FF2, Fmoc-Glu(OtBu)-Lys(Boc)-Asn(Trt)-Glu(OtBu)-Gln(Trt)$\mathrm{Glu}(\mathrm{OtBu})-\mathrm{Leu}-\mathrm{Leu}-\mathrm{Glu}(\mathrm{OtBu})-\mathrm{Leu}-\mathrm{OH}, \mathrm{MW}$ (monoisotopic) 2274.22, (average) 2275.76; FF3, $\mathrm{H}_{2} \mathrm{~N}$-Asp(OtBu)Lys(Boc)-Trp(Boc)-Ala-Ser(tBu)-Leu-Trp(Boc)-Asn(Trt)Trp(Boc)-Phe-CONH ${ }_{2}$, MW (monoisotopic) 2105.10, (average) 2106.50. Milli-Q water was used in all preparations. Acetonitrile (ACN) and N,N-dimethylformamide (DMF) were from Mallinkrodt Baker (Phillipsburg, NJ). Matrix compounds and inorganic salts were obtained from Sigma Aldrich (St. Louis, MO) and used without further purification.

\section{General Procedures for MALDI Sample Preparation}

For all experiments, peptide fragments were first dissolved to a concentration of $10 \mathrm{mg} / \mathrm{mL}$ (wt/vol) in DMF; these stock solutions were then diluted 100-fold into matrix solutions except where otherwise noted. Matrix solutions were prepared as saturated solutions and used within 1 day. When salts were used in sample preparations, a stock solution was prepared at 100 $\mathrm{mg} / \mathrm{mL}$ (wt/vol) in water and diluted 10-fold into the appropriate matrix solution to give a final matrix/salt solution which was saturated with respect to matrix and $10 \mathrm{mg} / \mathrm{mL}$ (wt/vol) with respect to salt.

\section{Optimized Sample Preparation for Protected Peptides}

The protected peptide to be analyzed is dissolved in DMF to a concentration of $10 \mathrm{mg} / \mathrm{mL}$ (wt/vol). A matrix stock solution is prepared by dissolving dithranol (CAS\# 1143-38-0) into ACN:water (1:1, vol/vol). $\mathrm{CsCl}$ is then prepared as a separate stock solution at 100 $\mathrm{mg} / \mathrm{mL}$ (wt/vol) in water and diluted 10-fold into the matrix solution. For sample analysis, the peptide solution is diluted 100-fold into the $\mathrm{CsCl} /$ dithranol solution and vortexed briefly; $1 \mu \mathrm{L}$ of this solution is then aliquoted onto a stainless steel target plate and allowed to dry at ambient temperature and pressure before MALDI sample analysis.

\section{MALDI Sample Analysis}

MALDI mass spectra were collected using a Bruker Microflex LRF MALDI mass spectrometer (Bruker Daltonics, Billerica, MA). Samples were analyzed in the linear positive ion mode, with 500 laser shots collected at random across each sample spot and summed using the automated sample collection mode. All spectra were processed by Savitzky-Golay smoothing and baseline subtraction.

\section{Results and Discussion}

Before the start of this work, it was apparent that conventional sample preparations for MALDI analysis of protected peptides left much to be desired. Mass spectra were difficult to collect and interpret due to poor sample spot morphology and sluggish ionization, and were usually accompanied by significant fragmentation. In the protected peptides under analysis the net result was that it was difficult to distinguish between bona fide synthetic (notably des-trityl and des- $t \mathrm{Bu}$ ) impurities and artifactual, source-induced fragmentation in the mass spectrometer.

We first reasoned that a screen of different matrices might uncover one more effective for the protected peptides under investigation. For instance, it is well known that sinapinic acid (SA) [10] is more suitable for large proteins than $\alpha$-cyano-4-hydroxycinnamic acid (4HCA) [11] or 2,5-dihydroxybenzoic acid (DHB) [12], and basic or charge-neutral matrices are more appropriate for oligonucleotide analysis [9, 13]. This latter point was especially noteworthy; it seemed reasonable 
Table 1. Summary of MALDI matrix compounds screened in FF1 analysis

\begin{tabular}{lc}
\hline \multicolumn{1}{c}{ Matrix } & Results $^{\mathrm{a}}$ \\
\hline \hline$\alpha$-Cyano-4-hydroxycinnamic acid (HCA) & + \\
2,5-Dihydroxybenzoic acid (DHB) & + \\
Sinapinic acid (SA) & - \\
2-(4-Hydroxyphenylazo)benzoic acid (HABA) & + \\
Dithranol & ++ \\
2-Amino-4-methyl-3-nitropyridine (3-ANP) & - \\
2-Amino-4-methyl-5-nitropyridine (5-ANP) & - \\
Pyrene & - \\
Anthracene & - \\
\hline
\end{tabular}

aKey: (-) no signal obtained, $(+)$ some signal obtained, with significant fragmentation, $(++)$ strong molecular ion signal obtained, with little fragmentation.

that if the fragmentation of protected peptides using classical peptide/protein MALDI matrices was due to the acidity of the matrix compound, then a less-acidic matrix would address this chemical incompatibility just as is the case for acid-labile oligonucleotides. In addition, the physicochemical similarity of protected peptides to hydrophobic polymers led us to consider that 2-(4-hydroxyphenylazo)benzoic acid (HABA) [14, 15], pyrene, or anthracene [16] might be appropriate for their analysis as well, just as these matrices have been reported to be useful for MS analysis of polymers. It should be emphasized, however, that the functional groups commonly present in peptides (even with protecting groups intact) impose a further complication over relatively nonfunctionalized polymers such as polystyrene, polyisoprene, and polybutadiene. As a result, all-trans-retinoic acid (RTA) and trans-3-indoleacrylic acid (IAA) [16] - two matrices also commonly used in MS analysis of polymers-were excluded from the present study owing to the reactivity of these matrices toward proteinaceous amine and thiol functionalities via conjugate addition reactions.

Our initial matrix screen was performed using FF1 (see Experimental section for structures) as a test analyte. The matrices selected in this study were chosen based on both historical success and electronic properties. HCA, SA, and DHB are the most widely-used matrices for peptide and protein analysis (vide supra), although DHB has found some application in polymer analysis [17]. HABA, pyrene, anthracene, and dithranol [18] were selected based on their well-known applicability in polymer analysis. It is noteworthy that pyrene and anthracene were also of interest due to their chargeneutral character, toward the end of investigating whether partial deprotection in MALDI analysis is due to acidic deprotection by carboxy-functionalized matrices. 2-Amino-4-methyl-3-nitropyridine (3-ANP) and 2-amino-4-methyl-5-nitropyridine (5-ANP) were also investigated, owing to their demonstrated utility in oligonucleotide analysis [9]. The FF1 analyte was selected for this screen because it represents a typical "real world" protected peptide; it has no basic functional groups, is of a reasonable size (16 amino acid residues), and is known to undergo variable degrees of detritylation at the His-1 side-chain during MALDI analysis (vide infra), though it is unclear whether this fragmentation occurs during sample preparation, ionization, or mass analysis.

To closely mimic a typical production sampling, a stock solution of FF1 was prepared in DMF and diluted 100-fold into matrix solutions, all prepared as saturated solutions in ACN:water (1:1, vol/vol). It should be emphasized that nonvolatile solvents are commonly known to be deleterious for MS analysis, and in the case of MALDI might lead to poorly-formed crystals on the sample target. Nonetheless, this sample preparation

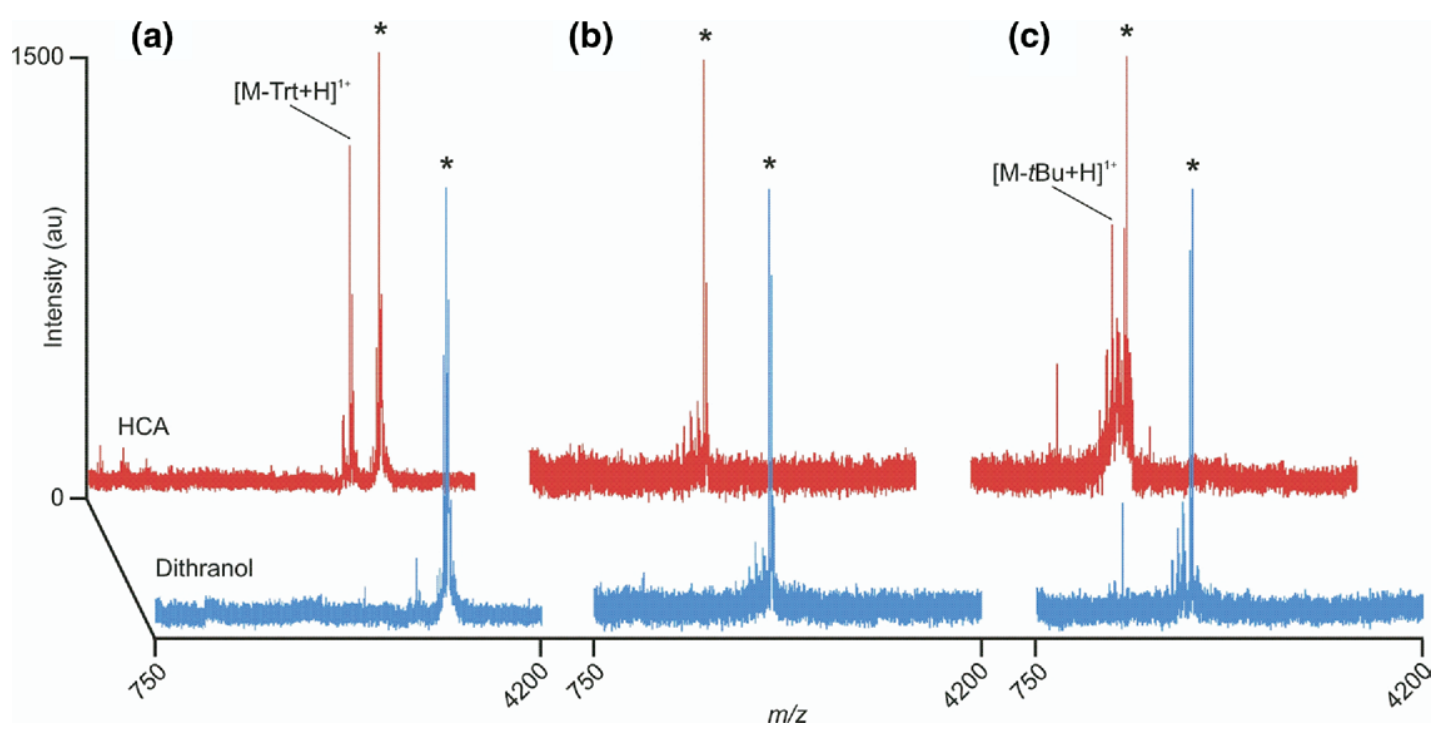

Figure 1. MALDI mass spectra of FF1 (a), FF2 (b), and FF3 (c) using HCA (red) and dithranol (blue) matrices. Asterisks denote molecular ions or $\mathrm{Na}^{+} / \mathrm{K}^{+}$adducts. 

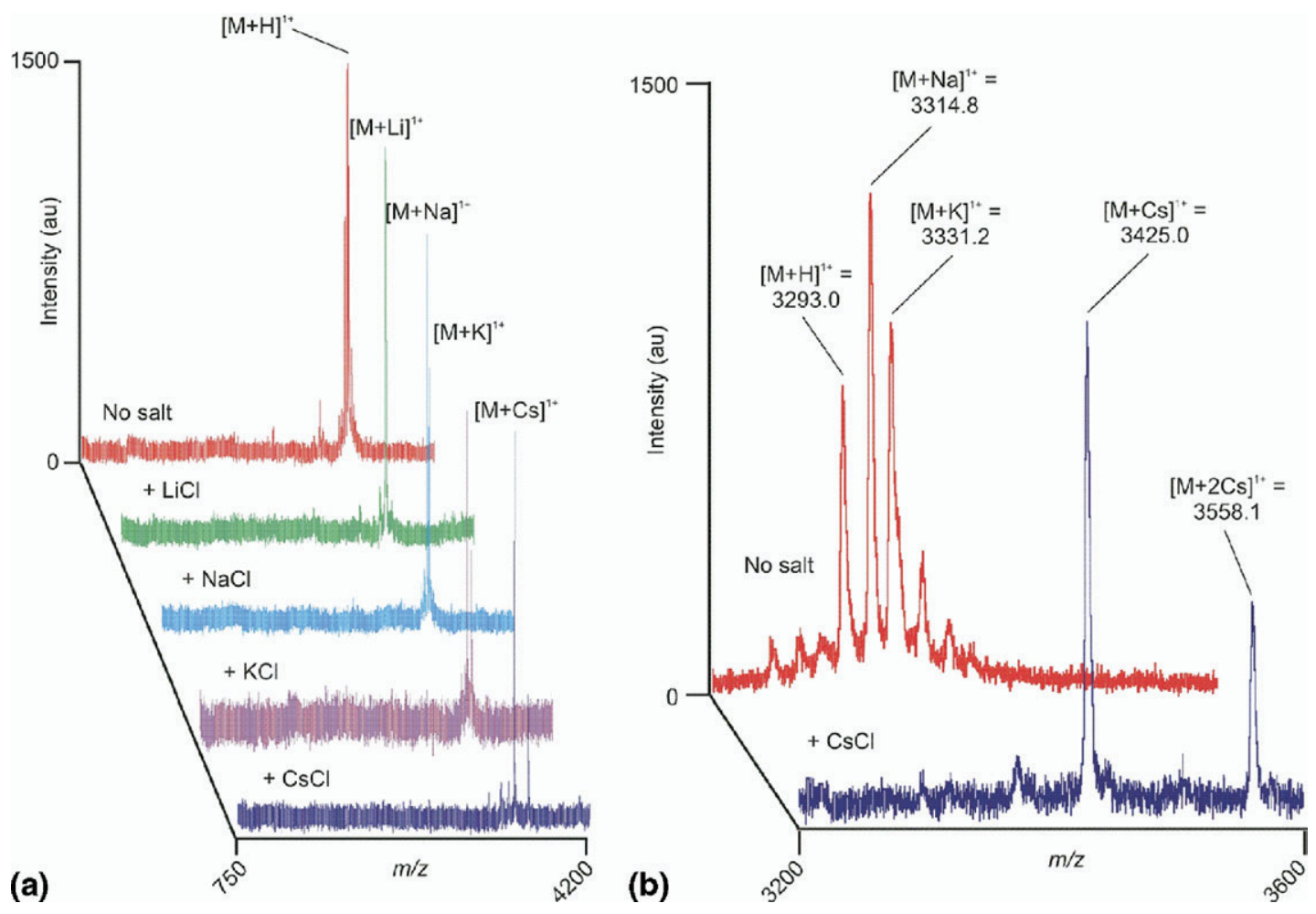

Figure 2. (a) Stacked MALDI mass spectra of FF1, prepared from a dithranol matrix without salt or with $10 \mathrm{mg} / \mathrm{mL}$ salt additives. (b) Expanded region of salt-free and CsCl-doped samples showing multiple cationized salt adducts arising from adventitious salt contamination.

was used because the goal of the present study is to provide a robust method useful in a typical production setting, wherein DMF is a near-generally applicable solvent for protected peptides. From the results of this initial matrix screen, it was clear that these matrices could be classified into three categories, with (1) no signal obtained, (2) some signal obtained, albeit with extensive fragmentation, or (3) the molecular ion was the predominant signal obtained, with little fragmentation. By far the most efficacious matrix was dithranol, which consistently afforded the easiest to obtain signals and the least fragmentation (Table 1). In most other cases-HCA, DHB, and HABA excepted-virtually no signal was obtained, and with HCA and DHB, significant fragmentation was observed.

To determine if dithranol would afford improved signal quality for more than one protected peptide analyte, MALDI spectra were obtained on Fuzeon fragments 1, 2, and 3 (FF1, FF2, and FF3) using dithranol and HCA as matrices. In all cases, dithranol provided significantly less source-induced fragmentation, despite the need for higher laser intensity to effect ionization (Figure 1). It is noteworthy that the signal-to-noise ratio of the molecular ions is comparable for dithranol and HCA, thus minimization of fragmentation is not accompanied by a loss in overall ionization intensity. One possible explanation for this difference is the significantly decreased acidity of dithranol $(\mathrm{pKa}=7.16 \pm$ $0.20)$ compared with HCA (pKa $=1.17 \pm 0.31)$ [19].
However, the well-known empirical nature of matrix performance, as well as the failure of the basic (3-ANP and 5-ANP) and uncharged (pyrene and anthracene) matrices underscore the point that other contributory factors are determinative of matrix performance in protected peptide analysis.

While the practical benefits of dithranol as a matrix in MALDI analysis of these protected peptides are clear, two outstanding issues motivated further optimization of this method. First, in many cases the crystalline morphology of the dried sample spots on the MALDI target is highly heterogeneous, thereby complicating automated sample collection; this is an important consideration in a high-throughput analytical support laboratory, wherein manual laser "navigation" is not a viable option. Second, interpretation of these spectra is occasionally difficult and requires knowledge of the various commonly-observed salt adducts obtained in MALDI. Toward the end of providing a generalized protocol accessible to the non-specialist, we sought to simplify this microheterogeneity to yield more easilyinterpreted spectra.

The addition of salts has been reported to aid in MALDI analysis of polymers devoid of functional groups by facilitating ionization under mild conditions $[16,17]$. A priori, this was a reasonable expedient to apply in the present application; synthetic peptides are in many cases homologous to hydrophobic polymers with few, if any, "handles" for ionization, and are 
marked by similar solubility profiles. We first attempted to use transition-metal salts derived from $\mathrm{Ag}^{\mathrm{I}}$ and $\mathrm{Cu}^{\mathrm{II}}$, but these quickly proved totally ineffective, and in fact nearly abolished ionization (data not shown). Moreover, the spectra obtained were dominated by unidentified low molecular weight species, likely either salt/matrix clusters or oxidatively decomposed samples (a likely possibility given that these metal salts are commonly used oxidants in peptide chemistry). We therefore considered that given the ease with which analytes, notably protected peptides, ionize as $\mathrm{Na}^{+}$or $\mathrm{K}^{+}$adducts, nonreactive alkali salts could prove efficacious as additives in the matrix solution. A screen of $\mathrm{LiCl}, \mathrm{NaCl}, \mathrm{KCl}$, and $\mathrm{CsCl}$ (added to the matrix solution at $10 \mathrm{mg} / \mathrm{mL}$ ) was therefore performed using FF1 as a test analyte, with all four salts yielding the expected cation adduct as the predominant singly-charged ion species (Figure 2a).

Two important observations were made from this experiment. First, the usual presence of multiple (typically ca. 5) cationized salt adducts is minimized through these intentional salt additions. As a result, spectra are much simpler to interpret, with automated peak picking algorithms identifying a single major species with an easily-assignable identity. It is noteworthy that in all salt additions, a singly-charged double-cation adduct was observed as a minor species. This is presumably the charge-neutral C-terminal carboxylate salt, and is most apparent in samples analyzed with added $\mathrm{CsCl}$. Second, the crystalline morphology of these samples on the MALDI target is significantly more homogeneous than without salt additions, which is likely due to the crystallization of added salts during sample spot evaporation. However, it is unclear whether these crystals are essentially the parent salts crystals interspersed within a dried peptide film, or rather a co-crystal lattice composed of matrix, analyte, and the exogenous salt additive. Whereas automated sample collection software was problematic in the case where the spot surface was marked by a few large monoliths or a film with most analyte on the outside rim, the addition of some of these salts results in an evenly "peppered" sample surface that allows for easy data acquisition.

Of the four salt additives, $\mathrm{CsCl}$ is the preferred additive for several reasons. Whereas $\mathrm{Na}^{+}$and $\mathrm{K}^{+}$ adducts are commonly found in comparable abundance due to trace amounts of these elements in solvents and glassware, $\mathrm{Cs}^{+}(+132.9)$ adducts are entirely due to intentional doping of the sample being analyzed. As a result, the correct $\mathrm{Cs}^{+}$adduct can be unambiguously identified with high confidence and without manual intervention, a key consideration in high throughput quality control laboratory settings (Figure 2b). Another noteworthy observation is the salt-dependent variation in crystalline morphology of the sample spot on the MALDI target surface. $\mathrm{LiCl}$ resulted in a glassy surface with few noticeable crystals, while $\mathrm{NaCl}$ gave large crystals where analyte was concentrated, with little signal obtained elsewhere on the target surface.
In contrast, $\mathrm{KCl}$ and $\mathrm{CsCl}$ produced homogeneous spot surfaces with even analyte distributions. For these reasons-vastly reduced heterogeneity in cationized salt adducts and homogeneous crystalline morphology on the sample target- $\mathrm{CsCl}$ is the preferred additive for protected peptide analysis by MALDI.

In a typical manufacturing setting, sample quantities are not limiting as is the case in proteomics applications; a sample peptide concentration of $10 \mathrm{mg} / \mathrm{mL}$ is therefore a reasonable concentration for sample solutions. However, protected peptides are often marked by gross insolubility, and within a research setting it can often be expected that sample quantities will be far more limiting than on a manufacturing scale. Considering that salt additions are commonly acknowledged to give rise to signal suppression in MS analysis [2022], we performed a sensitivity study to investigate whether the addition of $\mathrm{CsCl}$ to samples would have a detrimental effect on sensitivity. For this experiment, the FF1 stock solution was serially diluted in 10-fold increments to $1.0,0.1,0.01$, and $0.001 \mathrm{mg} / \mathrm{mL}$ before 100-fold dilution into dithranol or dithranol/ $\mathrm{CsCl}$ solutions. Even at the $1 \mu \mathrm{g} / \mathrm{mL}$ starting concentration, the same improvement in signal quality is discernable, with only 3 fmol analyte aliquoted onto the MALDI target (Figure 3).

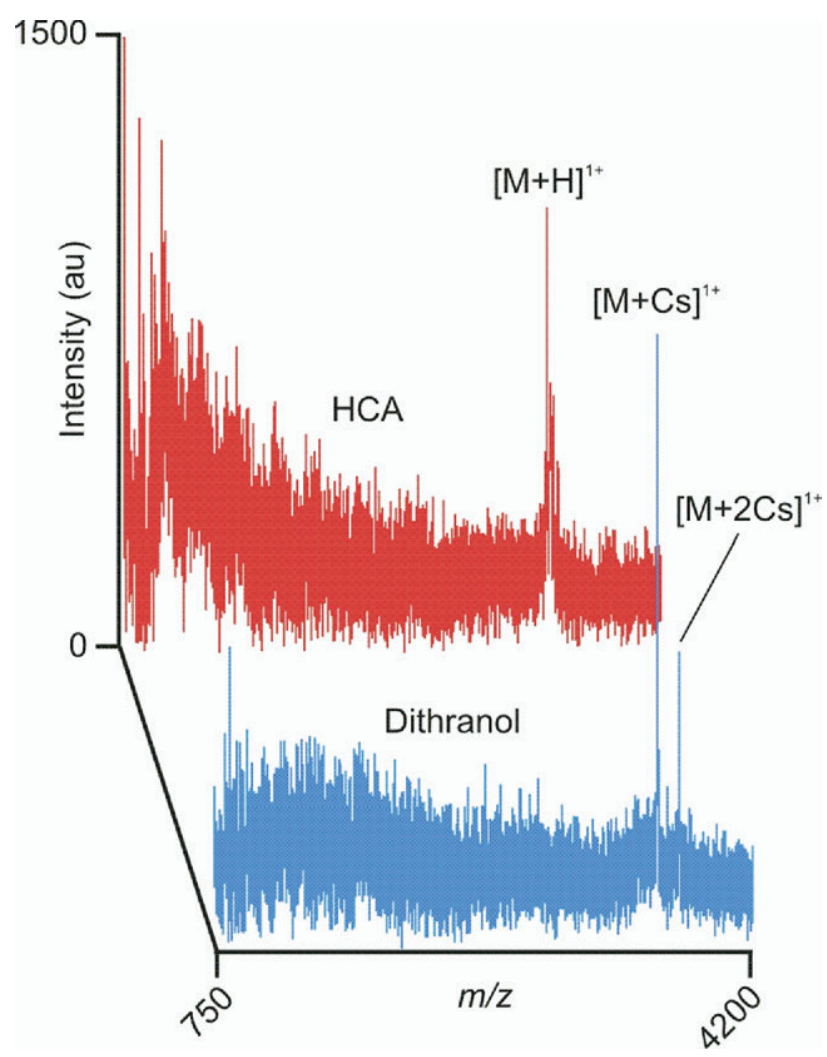

Figure 3. MALDI mass spectra of FF1 using dithranol (red) and dithranol/CsCl (blue) matrices at 10,000-fold dilution of original peptide stock solution, with each sample spot containing $3 \mathrm{fmol}$ analyte. 


\section{Conclusions}

MS analysis of protected peptides is an analytical challenge for several interrelated reasons. With all functional groups masked by hydrophobic protecting groups, this class of compounds is often sparingly soluble, requiring the use of solvents not commonly associated with sample preparation for MS analysis. The chemical lability of these functional groups imposes an additional complication, as source-induced fragmentation can be difficult to discern from genuine sample impurities. The intrinsic difficulty in MS analysis of non-ionizable compounds is a further obstacle to obtaining clean, easily interpreted mass spectra. In this report, dithranol has been shown to be an optimal matrix for three "real world" protected peptide samples, producing significantly less fragmentation in collected mass spectra. The addition of $\mathrm{CsCl}$ to the dithranol matrix solution is a further improvement, resulting in improved crystalline morphology on the MALDI sample target, as well as easily-interpreted mass spectra through the generation of unambiguous $\mathrm{Cs}^{+}$adducts, all without detriment to sensitivity. We envisage that these tactics will find use among investigators in process development and manufacturing settings, where facile, high-resolution MS analysis of protected peptide intermediates is a concern of primary importance.

\section{Acknowledgments}

The authors acknowledge Paul Bury for expert technical assistance, Dr. Grant Boldt (University of Oxford) for pKa calculations, and Joan Jorden for encouragement in this project. Matt Sweeney (PDL BioPharma) reviewed this manuscript and provided helpful comments.

\section{References}

1. Bruckdorfer, T.; Marder, O.; Albericio, F. From Production of Peptides in Milligram Amounts for Research to Multi-Ton Quantities for Drugs of the Future. Curr. Pharm. Biotech. 2004, 5, 29-43.

2. Bray, B. L. Large-Scale Manufacture of Peptide Therapeutics by Chemical Synthesis. Nat. Rev. Drug Discov. 2003, 2, 587-593.

3. Stults, J. T. Matrix-Assisted Laser-Desorption Ionization MassSpectrometry (MALDI-MS). Curr. Opin. Struct. Biol. 1995, 5, 691-698.
4. Skelton, R.; Dubois, F.; Zenobi, R. A MALDI Sample Preparation Method Suitable for Insoluble Polymers. Anal. Chem. 2000, 72, 17071710 .

5. Zhang, H.; Zhang, C.; Lajoie, G.; Yeung, K. K.-C. Selective Sampling of Phosphopeptides for Detection by MALDI Mass Spectrometry. Anal. Chem. 2005, 77, 6078-6084.

6. Norris, J. L.; Porter, N. A.; Caprioli, R. M. Mass Spectrometry of Intracellular and Membrane Proteins Using Cleavable Detergents. Anal. Chem. 2003, 75, 6642-6647.

7. Ekstrom, S.; Ericsson, D.; Onnerfjord, P.; Bengtsson, M.; Nilsson, J.; Marko-Varga, G.; Laurell, T. Signal Amplification Using "Spot on-aChip" Technology for the Identification of Proteins via MALDI-TOF MS. Anal. Chem. 2001, 73, 214-219.

8. Distler, A. M.; Allison, J. Improved MALDI-MS Analysis of Oligonucleotides through the Use of Fucose as a Matrix Additive. Anal. Chem. 2001, 73, 5000-5003.

9. Fitzgerald, M. C.; Parr, G. R.; Smith, L. M. Basic Matrices for the Matrix-Assisted Laser-Desorption Ionization Mass-Spectrometry of Proteins and Oligonucleotides. Anal. Chem. 1993, 65, 3204-3211.

10. Beavis, R. C.; Chait, B. T. Cinnamic Acid Derivatives as Matrices for Ultraviolet Laser Desorption Mass Spectrometry of Proteins. Rapid Commun. Mass Spectrom. 1989, 3, 432-435.

11. Beavis, R. C.; Chaudhary, T.; Chait, B. T. $\alpha$-Cyano-4-Hydroxycinnamic Acid as a Matrix for Matrix-Assisted Laser Desorption Mass Spectrometry. Org. Mass Spectrom. 1992, 27, 156-158.

12. Strupat, K.; Karas, M.; Hillenkamp, F. 2,5-Dihydroxybenzoic Acid: A New Matrix for Laser Desorption-Ionization Mass Spectrometry. Int. J. Mass Spectrom. Ion Processes. 1991, 111, 89-102.

13. Nordhoff, E.; Kirpekar, F.; Roepstorff, P. Mass Spectrometry of Nucleic Acids. Mass Spectrom. Rev. 1996, 15, 67-138.

14. Montaudo, G.; Montaudo, M. S.; Puglisi, C.; Samperi, F. 2-(4Hydroxyphenylazo)-Benzoic Acid: A Solid Matrix for Matrix-assisted Laser Desorption/Ionization of Polystyrene. Rapid Commun. Mass Spectrom. 1994, 8, 1011-1015.

15. Liu, H. M. D.; Schlunegger, U. P. Matrix-Assisted Laser Desorption/ Ionization of Synthetic Polymers with Azo Compound Matrices. Rapid Commun. Mass Spectrom. 1996, 10, 483-489.

16. Macha, S. F.; Limbach, P. A.; Hanton, S. D.; Owens, K. G. Silver Cluster Interferences in Matrix-Assisted Laser Desorption/Ionization (MALDI) Mass Spectrometry of Nonpolar Polymers. J. Am. Soc. Mass Spectrom. 2001, 12, 732-743.

17. Pastor, S. J.; Wilkins, C. L. Analysis of Hydrocarbon Polymers by Matrix-Assisted Laser Desorption/Ionization-Fourier Transform Mass Spectrometry. J. Am. Soc. Mass Spectrom. 1997, 8, 225-233.

18. Belu, A. M.; DeSimone, J. M.; Linton, R. W.; Lange, G. W.; Friedman, R. M. Evaluation of Matrix-Assisted Laser Desorption Ionization Mass Spectrometry for Polymer Characterization. J. Am. Soc. Mass Spectrom. 1996, 7, 11-24.

19. pKa values were calculated using Chemsketch version 5.0 (Advanced Chemistry Development, Toronto, Canada).

20. Smirnov, I. P.; Zhu, X.; Taylor, T.; Huang, Y.; Ross, P.; Papayanopoulos, I. A.; Martin, S. A.; Pappin, D. J. Suppression of $\alpha$-Cyano-4Hydroxycinnamic Acid Matrix Clusters and Reduction of Chemical Noise in MALDI-TOF Mass Spectrometry. Anal. Chem. 2004, 76, 2958 2965.

21. Fukuzawa, S.; Asanuma, M.; Tachibana, K.; Hirota, H. On-Probe Sample Preparation without Washes for Matrix-Assisted Laser Desorption/Ionization Mass Spectrometry Using an Anion Exchange Medium. Anal. Chem. 2005, 77, 5750-5754.

22. Winston, R. L.; Fitzgerald, M. C. Concentration and Desalting of Protein Samples for Mass Spectrometry Analysis. Anal. Biochem. 1998, 262, 83-85. 